\title{
Identified gaps and opportunities in perinatal healthcare delivery for women in treatment for opioid use disorder.
}

\author{
Karen Alexander \\ Thomas Jefferson University \\ Vanessa Short \\ Thomas Jefferson University \\ Megan Gannon \\ Thomas Jefferson University \\ Neera Goyal \\ Thomas Jefferson University

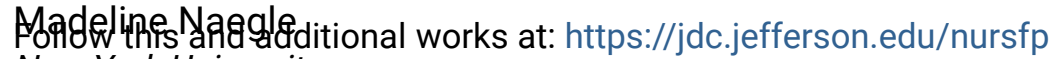 \\ Now York University \\ Part of the Nursing Commons \\ Let us know how access to this document benefits you
}

\section{Recommended Citation}

Alexander, Karen; Short, Vanessa; Gannon, Megan; Goyal, Neera; Naegle, Madeline; and Abatemarco, Diane J, "Identified gaps and opportunities in perinatal healthcare delivery for women in treatment for opioid use disorder." (2020). College of Nursing Faculty Papers \&

Presentations. Paper 105.

https://jdc.jefferson.edu/nursfp/105

This Article is brought to you for free and open access by the Jefferson Digital Commons. The Jefferson Digital Commons is a service of Thomas Jefferson University's Center for Teaching and Learning (CTL). The Commons is a showcase for Jefferson books and journals, peer-reviewed scholarly publications, unique historical collections from the University archives, and teaching tools. The Jefferson Digital Commons allows researchers and interested readers anywhere in the world to learn about and keep up to date with Jefferson scholarship. This article has been accepted for inclusion in College of Nursing Faculty Papers \& Presentations by an authorized administrator of the Jefferson Digital Commons. For more information, please contact: JeffersonDigitalCommons@jefferson.edu. 


\section{Authors}

Karen Alexander, Vanessa Short, Megan Gannon, Neera Goyal, Madeline Naegle, and Diane J Abatemarco 
2 Introduction

3

4

In the last two decades, the rate of pregnancies affected by opioid use disorder (OUD) in the United States has quadrupled ${ }^{1,2}$. Women with OUD often enter prenatal care later in pregnancy and stay longer in the hospital after birth compared to women without $\mathrm{OUD}^{3}$, and many also lack traditional support networks of family and friends ${ }^{4,5}$. In addition, a newborn exposed to intrauterine opioids may require medically supervised treatment for neonatal opioid withdrawal syndrome (NOWS) for several weeks after birth, a condition that is associated with significant neonatal morbidity and prolonged hospital stays ${ }^{6}$. The perinatal period, defined as the weeks immediately before, during and after the birth of a newborn, is therefore challenged by a structure of hospitalizations, multiple healthcare providers, and maintenance of OUD treatment.

The American College of Obstetrics and Gynecology (ACOG) recommends comprehensive perinatal healthcare delivery for women with OUD to ensure high-quality, safe care ${ }^{7}$, yet there is little documented in the literature as to the structure and components necessary to achieve this goal. In the general perinatal population, personalized approaches to perinatal care can improve delivery, contraception, and breastfeeding outcomes $^{8-10}$, as well as increasing a woman's health literacy ${ }^{11,12}$. Perinatal engagement strategies incorporated into standard care are also associated with increased quality of patient-provider interactions through improved patient activation ${ }^{13}$. Women with OUD are known to benefit from personalized prenatal care, demonstrating increased adherence to prenatal visits through a model which tailors support and education to the individual needs of substance exposed pregnancies ${ }^{14}$. Furthermore, tailored breastfeeding support has resulted in higher breastfeeding rates among substance-exposed women and infants ${ }^{15}$.

Despite the importance of personalized care, especially among high-risk populations ${ }^{16}$, little is known about how women with OUD perceive perinatal healthcare, including any efforts to prepare them for the birth and newborn healthcare experiences. 
1 However, pregnant women with OUD ${ }^{17}$ and women with infants diagnosed with NOWS ${ }^{18}$ do

2 report barriers to effective communication with their healthcare providers. Gathering

3 information on the perceptions of care could help inform a redesign of perinatal healthcare

4 for women with OUD by identifying unmet needs. Therefore, the aim of this study was to

5 identify and describe perceptions of the birth and newborn healthcare experiences among a

6 population of women in treatment for OUD in order to improve the delivery of perinatal

7 healthcare.

\section{Methods}

A qualitative study using focus groups with a convenience sample of participants recruited from a perinatal OUD treatment center was conducted. Qualitative thematic analysis methodology was used to sort and analyze the data through six steps: familiarization, generating initial codes, searching for themes, reviewing themes, defining and naming themes, and reporting themes ${ }^{19}$. The authors followed COnsolidated criteria for REporting Qualitative research (COREQ) principles to ensure comprehensive reporting of findings. This study was approved by the appropriate local institutional review boards.

\section{Study population and Setting}

All study participants were receiving comprehensive behavioral and physical health support and care plus pharmacotherapy (methadone) for OUD from a single outpatient treatment center. Study inclusion criteria were (1) 18 years or older; (2) receiving treatment for OUD; and (3) English speaking. All participants had legal guardianship of a child younger than 2 years of age. Recruitment continued until thematic saturation occurred, when no new data emerged.

\section{Recruitment and data collection}

In April 2019, counselors and front desk staff at the treatment center identified women who had a child less than 2 years of age. The identified women were given a brief 
1 description of the study and directed to speak to the principal investigator in a private space

2 if they were interested in participation. Those who expressed interest in participating were

3 then scheduled, according to their stated availability, to attend one of four separate focus group sessions over the subsequent month. Participants provided written informed consent prior to the sessions. Focus groups were utilized due to the reported comfort level of participants in a group setting as compared to one on one interviews, as a result of prior experience with research of this type at the center. not involved in the clinical care of study participants or the care of participants' children. Focus groups lasted on average 70 minutes and were audio-recorded. Focus groups were semi-structured and the discussion guide was based on the aims of the study, with openended questions and follow-up prompts to engage participants in a fluid discussion (Table 1). A survey which assessed demographics and perceptions of social support in the perinatal period was also administered. After completion of the focus group, $\$ 50.00$ was provided to each participant in the form of a ClinCard, a standard payment mechanism for distributing incentives to research participants.

\section{Data Analysis}

The audio recordings from each of the focus group sessions were transcribed verbatim. Following the last focus group session, the research team met to discuss data saturation and begin analysis. Saturation was determined after repetition had developed and no new information was obtained, which was agreed upon by both researchers (KA, VS).

22 The transcripts were analyzed using qualitative thematic analysis methods ${ }^{19}$ by a team of researchers with training in qualitative methods (KA, VS, MG). The process included generating initial codes, searching, reviewing, defining and naming subthemes, and identifying basic and global themes. Transcripts were initially reviewed independently with each researcher individually coding the transcripts. Then the team of researchers (KA, VS) 
1 met as a group to discuss the individually identified codes, merging similar ideas and

2 separating out unrelated constructs into discrete codes. These codes were then grouped

3 into two thematic patterns: 1) perceptions of preparation for the birth and newborn

4 healthcare experiences, and 2) perceptions of support.

The themes which emerged regarding social support were analyzed using the conceptual framework of Leahy-Warren ${ }^{20-22}$. Leahy Warren extends social exchange theory ${ }^{23}$ to maternal-child health and identifies four elements of social support: emotional concern, instrumental aid, informational support, and appraisal support. All types of support were discussed, and descriptions of the maternal perceptions were categorized based on the type of support. The final analysis was sent to the team and one additional researcher (NG) to ensure supporting data and themes were clear and distinct from each other.

Participants did not comment or give feedback on the themes.

Results between 4 and 8 participants per group. Four individuals who consented to participate in the study did not attended their scheduled session due to childcare challenges, and one participant passed away prior to the start of the study. The mean age of participants was 31 years of age, and the average age of participants' most recently delivered child was 11 months of age. Participant demographics are displayed in Table 2. The two major themes, perceptions of preparation and perceptions of support, will be discussed below with their subthemes with representative quotes pertaining to each subtheme presented with a randomized participant number following the quote. Additional supporting quotations are available in Appendix A.

\section{Perceptions of preparation for the birth and newborn healthcare experiences}

\section{Birth: Sudden and Unexpected}

Women consistently mentioned the sudden and unexpected nature of their delivery. 
1 they lacked emotional preparation for the delivery. Women described situations where they

2 had to be emergently admitted to the hospital, or driven in someone's car when they were

3 almost to the point of delivering the baby. Women expressed that they felt there was no

4 way to prepare for the delivery, despite receiving prenatal care.

I wasn't prepared. Like I had her stuff or whatever, I was prepared as far as like her coming, but having her I was [emotionally], I was not prepared at all. (Participant 20)

Women recalled all types of deliveries, including emergency C-sections, and many reported being undertreated for the level of pain.

I know that one thing that I felt with the anesthesiologist- Like the level of pain that's normal, maybe there could be a conversation around that...And I don't know if it was because of my tolerance being high from, you know, using the opiates or whatever, but I felt like I felt everything. (Participant 11)

Women believed their opioid use disorder had contributed to their experience of pain, but they also felt that providers didn't listen to them. Many women felt it was important for healthcare providers to acknowledge their OUD and the steps they were taking in treatment. Women wished they had conversations about OUD treatment and how it may affect delivery, and attributed the lack of this discussion to the urgent nature of their birth.

\section{Birth: Connected to Recovery}

Some women reported that they entered treatment for OUD days before the delivery of their child. More than one women mentioned the need to defend their choice to be in treatment during pregnancy when they came to delivery (see Appendix A). Although they knew this was the best choice for them, they felt a need to justify and protect themselves among healthcare providers.

I said yes, my son was born with methadone in his system because I was on methadone when I was pregnant with him. The reasons why I was on it [are my] business and I'm not about to tell them. (Participant 6) 
A pregnancy while in OUD treatment felt significant and positive. Women also spoke

2 of birth as a tenuous and stressful time. Numerous stories were told of other women who

3 had not been able to adhere to an OUD treatment program or maintain custody of their

4 newborn because of re-initiating drug use.

Birth is traumatic for any woman, and so, some women, [treatment centers] push them so much they actually do relapse right after birth because they can't take it. (Participant 20)

OUD treatment was seen as a difficult journey, but a necessary positive step towards turning their life in a different direction. They understood the effects of OUD on their family connections, and they desired a different outcome for their recent child. Yet, women spoke of the drawbacks of methadone maintenance for OUD, including the side effects and general restrictions on their lives due to treatment structure. It was discussed that treatment centers had many rules to navigate, and medication caused concerns about the health of their baby.

Yes, if you are using, methadone is [a good option]. Methadone saved not only my life, but my baby's too. So regardless of how much pain and the side effects that it come with ... just imagine if we didn't have it. (Participant 18)

Women expressed guilt over prior choices related to drugs, and acknowledged the enormous effort it takes to enter treatment during pregnancy. It was stated that having a conversation with healthcare providers about the specifics of treatment and its unique effect on pregnancy would be welcomed, and may be useful to assuage fears about causing harm to the baby.

\section{Newborn: Lack of information}

All women in the study were prescribed and actively taking methadone for OUD. Many women spoke of their methadone dosage as high or low, and thought it would indicate the severity of neonatal opioid withdrawal syndrome (NOWS). Women in the group would interrupt the others in the group, trying to correct the information. 
But I was only on 160 milligrams. I know women who, I know one person, she was on 365, her daughter didn't go through anything. So like I was completely unprepared and I had to watch my daughter go through what she went through, really $\mathrm{f}^{\star *}$ ed me up because I knew nothing about that. (Participant 16)

Information about connections between dosages and length of stay circulated

6 informally. Preparation came in the form of talking to other women in treatment and

remembering their own previous experiences. Many women attended group therapy sessions and would hear stories about other babies going through withdrawal. These stories scared them without a context or framework for the bigger picture of NOWS.

Newborn: Forming their identity as a mother

Healthcare providers, mainly identified as nurses, were seen as the gatekeeper to forming an identity as a mother. It was discussed that some nurses allowed the women to perform caretaking behaviors that a typical mother would perform, but that some nurses would not. Women described nursing staff who would allow them privacy and the ability to be present with their child. In contrast, women also described nursing staff who would stand over them while they tried to hold their child, waiting for them to look like they may fall asleep.

perceived by the women as having a direct, negative effect on their newborn in treatment for NOWS.

\section{Perceptions of social support during birth and newborn hospitalizations}

Birth: Emotional Concern

Women expressed that emotional concern was not provided by all healthcare 
1 delivery. Women perceived that this was due to their history of drug use, and acknowledged

2 that this interaction was expected, had occurred previously in healthcare settings. Most women had found providers for their prenatal care that they felt provided emotional concern, but they knew they couldn't control who was assigned during delivery.

One woman related that her birth occurred without any support person,

6 without a partner or a family member. Some women had been labor support for each other in the delivery room, and others were able to have their mother or a sister with them. One woman mentioned having a doula present for her delivery as an emotional and instrumental support. A doula was able to teach ways of coping with pain, and explain procedures that were suggested by the healthcare providers. The doula was able to obtain a birthing ball, for example, or essential oils to provide holistic pain relief. It was perceived as a benefit to being in recovery that a doula could be involved in their care. people to help me with my pregnancy but my other [son]... I was on drugs with my last son. (Participant 3)

The labor support person was seen as trusted and knowledgeable resource that could act as a liaison on their behalf to the healthcare team, and someone who could act as an advocate.

Newborn: Instrumental aid

Nurses were seen as providers of instrumental support during the newborn hospitalization, teaching women how to care for their child in the context of medical needs. Nurses were identified as teaching feeding techniques, the scoring protocol for NOWS and techniques for soothing a newborn with withdrawal symptoms.

Women felt dependent on nurses in the neonatal intensive care unit (NICU) to teach them how to provide the best care for their child, since many of the children had 
1 special needs in terms of feeding. The women also felt that the child did not score as

2 well in their absence, and they appreciated any accommodations that could be made to 3 improve their ability to be at the bedside. One woman mentioned that she was able to room-in during the NOWS stay with her newborn for the duration of the treatment. She felt as if that improved her child's feeding ability from a bottle instead of needing a nasogastric tube, and that her newborn's scores improved if she was present. Most women who delivered at the affiliated hospital with the treatment center reported not being provided rooming-in accommodations.

Newborn: Informational and appraisal support

Maternal mothers $(n=12)$ and the baby's doctor $(n=6)$ were the most frequently identified sources of informational support in terms of newborn care as seen in Table 2. Although many women with substance use disorders are estranged from both the healthcare system and from family ${ }^{24}$, entry into OUD treatment during the perinatal period can prompt renewed communication with trusted sources of support. Maternal mothers were discussed as sources of information to explain newborn and medical care.

Those that did not have maternal support felt they were on their own in figuring out information. Women expressed fear about acting on information that was not valid for their particular situation. Women felt that they were entering a system where the boundaries were not clear, and healthcare providers differed on the guidance they provided.

When emotional support was assessed, women mentioned again that their own mother $(n=10)$ or their baby's father $(n=4)$ were the most frequently identified support sources. Although discussed, appraisal support, which relates to positive feedback on behavior was not always present from healthcare providers or external support sources. Feedback took the form of judgment related to the women's recovery and opioid use 
1 disorder and was directly communicated and indirectly perceived by the women during the newborn hospitalization. Women talked of responding to healthcare providers who treated them in a condescending manner:

I'd another experience with a nurse that was like talking down to me about my ... "Oh just don't use drugs again." Giving me like the whole speech like, that's not your job. That's not your job like, your job is my child not my, you know?

Women perceived a need to defend their recovery and felt as if they were on guard.

Rather than feeling supported in their parenting journey, some healthcare providers communicated that their past governed their current ability to be a mother to the newborn.

\section{Discussion}

1 This study explored how women with OUD experience the birth and newborn healthcare experiences, the results of which identified gaps and strengths of existing perinatal healthcare delivery to women with OUD in one institution. Women with OUD saw birth while in recovery as a positive experience, although many felt unprepared for the many possible ways the delivery could unfold. Although all participants were receiving care in an academic medical center which specializes in the care of women with OUD, women lacked information and received misinformation regarding how OUD affects their pregnancy, birth and their newborn. The data highlight existing issues in the delivery of perinatal healthcare to women with OUD, and present challenges that need to be addressed in order to provide quality care for women with OUD 25 .

Comprehensive perinatal OUD treatment includes both medication, behavioral therapy and care coordination services. The women in our study had access to this type of treatment, and previous qualitative studies show that women with OUD in other contexts find this type of healthcare integration essential to quality care ${ }^{26,27}$. Yet, the majority of women in the United States do not have access to comprehensive perinatal health care tailored for the complexities of a pregnancy with OUD. In 2015, less than a fourth of OUD treatment centers in the United States provided gender-specific care ${ }^{28}$, despite the $50 \%$ increase in the 
1 rate of women using opioids compared to $\operatorname{men}^{29,30}$. Although birth while in recovery was a

2 positive experience for the women in the study, in some areas of the country, this is not

3 possible or is inaccessible for the majority of women. A survey of Medicaid data in the

4 United States revealed that among women in the southern United States admissions to

5 medication-assisted treatment occurred at a rate of $30.6 \%$ compared to $54.2 \%$ in the

6 northeastern United States where this study occurred ${ }^{31}$. Despite their access to

7 comprehensive care, women in the study identified further areas of improvement

8 surrounding their preparation and support in the birth and newborn healthcare experiences. Many women reported misinformation from peers, and reported that their family or friends were the main sources of information regarding delivery and newborn care, despite consistent interaction with healthcare providers. The women desired more knowledge from trusted sources before delivery, and healthcare professionals were identified as trusted sources. Women stated that information provided by clinicians in real-time was perceived as emotionally supportive, and this is important as it is known that women with OUD report lower self-efficacy in their decisions to remain in recovery and maintain their health goals ${ }^{27}$. Appropriate support from healthcare providers independently impacts maternal confidence, self-efficacy and post-partum depression outcomes $22,24,32,33$. Reminding clinicians to engage in conversation with women, while offering them information and positive feedback specifically towards their choice for recovery creates a collaborative environment. Information from trusted sources can empower a woman and enable her to advocate for her own health, and operate as a full partner in her care ${ }^{13}$.

Women also reported inadequacy of pain coverage during birth, with women expressing fear that providers will not understand or may be biased in their treatment of their pain because of their OUD. There is limited current knowledge of intrapartum pain control management for women with OUD, but studies in the general population have demonstrated

26 that individuals with OUD can experience heightened pain (hyperalgesia) due to chronic exposure to opioids ${ }^{34,35}$. Further research is needed to develop standardized protocols 
1 which effectively management intrapartum and postpartum pain for women with OUD.

2 Standardized education for obstetric healthcare providers is also needed to decrease bias and judgement and improve the quality of pain management delivered.

Women desired participation in their newborn's care as they formed their identity as mothers, but many healthcare providers (specifically identified as nurses) did not permit them to perform typical maternal care of their newborn. This is consistent with both prior qualitative work with women with infants diagnosed with NOWS ${ }^{5,18}$, and a study of nurses in the neonatal intensive care unit regarding their interaction with mothers of infants with NOWS ${ }^{36}$. The nurses reported moral distress while for women with OUD and their children, and nurses were capable of assigning blame to the mother ${ }^{36}$ for the severity of withdrawal symptoms in the infant. An environment of judgement can be a barrier to effective communication with healthcare providers, and may effect teaching and learning of caregiving behavior.

All but one woman in this study reported visitation restrictions in caring for her newborn during hospitalization, and the women described those restrictions as an impediment to their caregiving, bonding and negatively affected withdrawal symptoms in their infant. One institution has demonstrated decreased lengths of stay for infants with NOWS ${ }^{37}$ and decreased use of pharmacologic treatment for NOWS ${ }^{38}$ through implementing a predictable schedule for infants with NOWS, coupled with rooming-in policies to increase maternal presence at the bedside. A focus on improving the preparation and support available to this population will require collaboration across teams of healthcare professionals and must include the perspectives of women with OUD.

There are several limitations to this study. First, the participants were recruited from a comprehensive OUD treatment center, affiliated with a large academic hospital OB-GYN department. Therefore, the perspectives of one context of delivery is most likely reflected in these results, potentially biasing the findings. In addition, we used a convenience sample of women who were willing to participate in focus groups during the day, potentially limiting 
1 responses to women who were not employed. The perspectives of women who have

2 experience with birth and neonatal hospitalizations outside our institution could also add

3 valuable knowledge. As with any qualitative data collection, the possibility of social

4 desirability is a potential concern, but the focus group format does increase comfort of some

5 participants with sharing their stories. Women in this particular treatment center are very

6 familiar with the other participants, their experiences, and feel largely comfortable revealing

7 details of their journey.

$8 \quad$ Women with OUD shared valuable perspectives in this study which will inform the

9 future delivery of perinatal healthcare. Comprehensive perinatal care for women with OUD

10 should empower the mother to care for herself and her baby through emphasizing a

11 partnership with the woman at the center of care. A focus on accessible, personalized care

12 for women with OUD may also improve crucial maternal and infant outcomes. 
1. Haight S, Ko J, Tong V, Bohm M, Callaghan W. Opioid Use Disorder Documented at Delivery Hospitalization — United States, 1999-2014. Morb Mortal Wkly Rep Surveill Summ. 2018;67:845-849.

2. Huang X, Keyes KM, Li G. Increasing Prescription Opioid and Heroin Overdose Mortality in the United States, 1999-2014: An Age-Period-Cohort Analysis. Am J Public Health. 2018;108(1):131-136.

3. Clemans-Cope L, Lynch V, Howell E, et al. Pregnant women with opioid use disorder and their infants in three state Medicaid programs in 2013-2016. Drug Alcohol Depend. 2019;195:156-163.

4. Benningfield MM, Arria AM, Kaltenbach K, et al. Co-occurring psychiatric symptoms are associated with increased psychological, social, and medical impairment in opioid dependent pregnant women. Am J Addict. 2010;19(5):416-421.

5. Cleveland L, Gill S. "Try Not to Judge": Mothers of Substance Exposed Infants. MCN: The American Journal of Maternal/Child Nursing. 2013;38(4):200-205.

6. Tolia VN, Patrick SW, Bennett MM, et al. Increasing Incidence of the Neonatal Abstinence Syndrome in U.S. Neonatal ICUs. N Engl J Med. 2015;372(22):21182126.

7. Krans EE, Campopiano M, Cleveland LM, et al. National Partnership for Maternal Safety: Consensus Bundle on Obstetric Care for Women With Opioid Use Disorder. Obstet Gynecol. 2019;134(2):365-375.

8. Rasouli M, AtashSokhan G, Keramat A, Khosravi A, Fooladi E, Mousavi SA. The impact of motivational interviewing on participation in childbirth preparation classes and having a natural delivery: a randomised trial. BJOG. 2017;124(4):631-639.

9. Otsuka K, Taguri M, Dennis C-L, et al. Effectiveness of a breastfeeding self-efficacy intervention: do hospital practices make a difference? Maternal and child health journal. 2014;18(1):296-306.

10. Smith E. Centering contraception: postpartum contraceptive choices of women enrolled in Centering group prenatal care versus traditional prenatal care. BMJ sexual \& reproductive health. 2018;44(2):103-108.

11. Roch G, Borges Da Silva R, de Montigny F, et al. Impacts of online and group perinatal education: a mixed methods study protocol for the optimization of perinatal health services. BMC Health Serv Res. 2018;18(1):382.

12. Logsdon MC, Davis D, Eckert D, et al. Feasibility of Two Educational Methods for Teaching New Mothers: A Pilot Study. Interactive journal of medical research. 2015;4(4):e20.

13. Ledford CJW, Sadler KP, Jackson JT, Womack JJ, Rider HA, Seehusen AB. Applying the chronic care model to prenatal care: Patient activation, productive interactions, and prenatal outcomes. Patient Educ Couns. 2018;101(9):1620-1623.

14. Sutter MB, Watson H, Bauers A, et al. Group Prenatal Care for Women Receiving Medication-Assisted Treatment for Opioid Use Disorder in Pregnancy: An Interprofessional Approach. J Midwifery Womens Health. 2019;64(2):217-224.

15. MacVicar S, Humphrey T, Forbes-McKay KE. Breastfeeding and the substanceexposed mother and baby. Birth. 2018;45(4):450-458.

16. Olivia Kim U, Barnekow K, Ahamed SI, et al. Smartphone-based prenatal education for parents with preterm birth risk factors. Patient Educ Couns. 2019;102(4):701-708.

17. Kuo C, Schonbrun YC, Zlotnick C, et al. A qualitative study of treatment needs among pregnant and postpartum women with substance use and depression. Subst Use Misuse. 2013;48(14):1498-1508. 
18. Cleveland L, Bonugli R. Experiences of Mothers of Infants with Neonatal Abstinence Syndrome in the Neonatal Intensive Care Unit. Journal of Obstetric, Gynecologic \& Neonatal Nursing. 2014;43(3):318-329.

19. Vaismoradi M, Turunen H, Bondas T. Content analysis and thematic analysis: Implications for conducting a qualitative descriptive study. Nurs Health Sci. 2013;15(3):398-405.

20. Leahy-Warren P, McCarthy G, Corcoran P. First-time mothers: social support, maternal parental self-efficacy and postnatal depression. J Clin Nurs. 2012;21(34):388-397.

21. Leahy-Warren P. First-time mothers: social support and confidence in infant care. $J$ Adv Nurs. 2005;50(5):479-488.

22. Leahy-Warren P, McCarthy G, Corcoran P. First-time mothers: social support, maternal parental self-efficacy and postnatal depression. J Clin Nurs. 2011;21(34):388-397.

23. House JS. Work, Stress and Social Support. Reading, MA: Addison-Wesley; 1981.

24. Cavaiola AA, Fulmer BA, Stout D. The Impact of Social Support and Attachment Style on Quality of Life and Readiness to Change in a Sample of Individuals Receiving Medication-Assisted Treatment for Opioid Dependence. Subst Abus. 2015;36(2):183-191.

25. ACOG Committee Opinion No. 742 Summary: Postpartum Pain Management. Obstet Gynecol. 2018;132(1):252-253.

26. O'Rourke-Suchoff D, Sobel L, Holland E, Perkins R, Saia K, Bell S. The labor and birth experience of women with opioid use disorder: A qualitative study. Women Birth. 2020.

27. Latuskie KA, Andrews NC, Motz M, et al. Reasons for substance use continuation and discontinuation during pregnancy: a qualitative study. Women and Birth. 2019;32(1):e57-e64.

28. SAMSHA. Clinical Guidance for Treating Pregnant and Parenting Women With Opioid Use Disorder and Their Infants. In: SAMSHA, ed. Rockville, MD: Substance Abuse and Mental Health Services Administration; 2018.

29. McHugh RK, Wigderson S, Greenfield SF. Epidemiology of Substance Use in Reproductive-Age Women. Obstet Gynecol Clin North Am. 2014;41(2):177-189.

30. McHugh RK, Votaw VR, Sugarman DE, Greenfield SF. Sex and gender differences in substance use disorders. Clin Psychol Rev. 2018;66:12-23.

31. Hand DJ, Short VL, Abatemarco DJ. Substance use, treatment, and demographic characteristics of pregnant women entering treatment for opioid use disorder differ by United States census region. J Subst Abuse Treat. 2017;76:58-63.

32. Shorey S, Chan SWC, Chong YS, He HG. Maternal parental self-efficacy in newborn care and social support needs in Singapore: a correlational study. J Clin Nurs. 2014;23(15-16):2272-2283.

33. Brown A. Maternal trait personality and breastfeeding duration: the importance of confidence and social support. J Adv Nurs. 2014;70(3):587-598.

34. Landau R. Post-cesarean delivery pain. Management of the opioid-dependent patient before, during and after cesarean delivery. Int J Obstet Anesth. 2019;39:105-116.

35. Safley RR, Swietlikowski J. Pain Management in the Opioid-Dependent Pregnant Woman. J Perinat Neonatal Nurs. 2017;31(2):118-125.

36. Maguire D, Webb M, Passmore D, Cline G. NICU nurses' lived experience: caring for infants with neonatal abstinence syndrome. Adv Neonatal Care. 2012;12(5):281-285.

37. Howard MB, Schiff DM, Penwill N, et al. Impact of parental presence at infants' bedside on neonatal abstinence syndrome. Hospital pediatrics. 2017;7(2):63-69. 
1 38. Wachman EM, Grossman M, Schiff DM, et al. Quality improvement initiative to improve inpatient outcomes for neonatal abstinence syndrome. $J$ Perinatol. 2018;38(8):1114-1122. 